\title{
Die behauptete und die gelebte Migrationskrise: der widersprüichliche Fall Italien
}

\author{
Felicitas Hillmann ${ }^{1,2}$ \\ ${ }^{1}$ Forschungsabteilung Regenerierung von Städten, IRS, Erkner, Germany \\ ${ }^{2}$ Institut für Stadt- und Regionalplanung, TU Berlin, Berlin, Germany \\ Correspondence: Felicitas Hillmann (felicitas.hillmann@leibniz-irs.de, hillmann@tu-berlin.de)
}

Received: 1 August 2018 - Revised: 30 July 2019 - Accepted: 19 September 2019 - Published: 2 December 2019

Kurzfassung. Italy, situated prominently middle of the Mediterranean Sea, has been confronted with migration and refugee issues for a long time. Within the European migration system it is a telling example of the way the issue of migration is dealt with more generally. After a prolonged phase of ignoring the existing exigencies of integration and regulation of the already present migrants on the territory, the country experienced a peak of arrivals of migrants on its coastlines in 2015/16. Since then, numbers of in-migration have been decreasing. Still, in the public discourse the idea of an invasion, e.g. mass immigration, continues. For many years the SPRAR system had contributed to tackling a situation of crisis by involving civil society and by reaching out for solutions beyond management. The article emphasizes that the migration crisis can be understood only when taking into account different realities and expectations. It further presents the case study of Genoa. Here, the crisis is mastered by normalisation and local action.

\section{Europas Migrationskrise in sozialgeographischer Perspektive}

Über kein Thema wurde im letzten Jahr in Deutschland so heftig, polarisierend und anhaltend debattiert wie über die Flüchtlingskrise (Vowe, 2016:435; Greck, 2018). Innenminister Horst Seehofer wetterte gegen die Flüchtlingspolitik von Angela Merkel und bezeichnete in der Talkshow von Sandra Maischberger den Kern der Migrationskrise als „Asyltourismus“. Er unterstellte damit, dass die Menschen, die nach Deutschland flüchten, sich wie alle anderen EUBürger auf dem Territorium bewegen könnten. Dabei besteht ein großer Teil der Krise dieser Menschen ja gerade darin, dass sie sich nicht frei bewegen können, sich aber dennoch von A nach B bewegen müssen. Ihre Mobilität unterliegt anderen Gesetzen als die von EU-Bürgern. Der Fall Italien bietet innerhalb des europäischen Migrationssystems ein außergewöhnlich zugespitztes Beispiel für den Umgang mit dieser europäischen „Migrationskrise“ ${ }^{\text {.1. }}$.

\footnotetext{
${ }^{1}$ Die hier gesetzten Anführungszeichen deuten die starke politische Aufladung des Themas an. Teile der kritischen Migrationsforschung sehen in der Verwendung dieser Begrifflichkeit bereits eine
}

Diese Krise entpuppt sich bei näherer Betrachtung als Bestandteil eines umfassenderen gesellschaftlichen Transformationsprozesses, der durch veränderte Mobilitäten und Migrationsmuster und verstärkte Ungleichheiten geprägt ist (Sheller, 2018). Dieser Transformationsprozess, so wird hier argumentiert, wird vor allem auch deshalb als krisenhaft empfunden, weil er den normativen Kern unserer gesellschaftlichen Verfasstheit berührt. Die Frage, wer wie mobil sein darf und wessen Menschenwürde schützenswert ist, ist eine ethische und hochpolitische Frage, die die Gesellschaft und Städte vor praktische, weil lokal zu bearbeitende Problemlagen, stellt. Momentan sind es vor allem die Städte, die angesichts der Dramatik der Migrationskrise handeln. Entgegen der Weisung der nationalen Regierungen nehmen sie Flüchtlinge in Seenot auf und schließen sich dazu in europaweiten Städtenetzwerken zusammen (als Beispiel sei hier die „Seebrücke“ genannt), die Bürgermeister vieler Städte bezie-

Übernahme von Mainstream-Ideologien, die othering betreiben und damit implizierten, dass „Migration und MigrantInnen“ als die Abweichung von der Norm angesehen würden. Während die Fachliteratur hier äußerst reflektiert ist, nutzen weite Teile der öffentlichen Debatte den Begriff ohne Vorbehalte. 
hen Position für MigrantInnen. Die Zuspitzung der Migrationskrise zeigt vor allem auch eines: dass disziplinäre und national ausgerichtete Erklärungsansätze nicht zum Verständnis und erst recht nicht zur Bearbeitung der Migrationskrise in der Praxis ausreichen. Was vielmehr benötigt wird, ist eine integrative Perspektive, die das Zusammenwirken unterschiedlicher Teilkrisen auf verschiedenen sozialräumlichen Ebenen in den Blick nimmt und vor allem auch der zeitlichen Dimension, d.h. dem Prozess der Herstellung der Krise, Beachtung schenkt. Dieser Ansatz, der davon ausgeht, dass Migration als räumliche Definitionsmacht auf verschiedenen Handlungsebenen wirkt und dass die Krise durch unauflösliche Widersprüche und radikale Handlungslogiken zwischen den verschiedenen Handlungsebenen produziert wird, wird hier als sozialgeographische Perspektive gefasst (Hillmann, 2016). Diesem Ansatz einer multiperspektivischen Betrachtung folgend, werden in diesem Beitrag zunächst unterschiedliche Blickwinkel auf die Krise beleuchtet (worin besteht die Krise und um wessen Krise handelt es sich?) (Abschnitt 2), dann wird das Fallbeispiel Italien und dessen institutionelles Krisenmanagement als Teil der Krisenproduktion beleuchtet, insbesondere auf Ebene der Städte (Abschnitt 3). Schließlich wird ein konkretes Fallbeispiel dargestellt, das zeigt, wie in der Praxis Gegenmodelle zur Migrationskrise entstanden und wie sehr diese auf eine Herstellung von Normalität als Gegengift zur Krise zielten (Abschnitt 4). ${ }^{2}$ Das Fazit bündelt die verschiedenen Argumentationsstränge und arbeitet den sozialgeographischen Gehalt der Migrationskrise heraus.

Die hier verwendeten empirischen Daten entstanden im Rahmen eines Projektes zum Umgang mit migrationsbezogener Vielfalt in europäischen Städten. Während vier Forschungsaufenthalten in den Jahren 2016 und 2017 wurden insgesamt 30 Interviews mit Stakeholdern der Zivilgesellschaft und der Kommune im Bereich Migration und Integration geführt, außerdem das hier vorgestellte Beispiel der Flüchtlingsunterbringung besucht ${ }^{3}$.

\section{Welche, vor allem: wessen Krise?}

Der Begriff der „Krise“ ist unscharf, eine einheitliche Definition existiert nicht. Ganz allgemein wird hierunter ,ei-

\footnotetext{
${ }^{2}$ Die hier präsentierten Befunde wurden im Rahmen des am Leibniz-Institut für Raumbezogene Sozialforschung (Erkner) durchgeführten Leitprojektes „UrbanReg - Urbane Regenerierungspraxen, Zuwanderung und die Produktion sozialräumlicher Ungleichheiten in europäischen Städten“ (2015-2018) erarbeitet. Die 30 Interviews wurden mit Verwaltungsmitarbeitern der Stadt Genua, Stakeholdern der Zivilgesellschaft sowie Wissenschaftlern geführt.

${ }^{3}$ Eine umfangreiche Betrachtung der italienischen, insbesondere der genuesischen Migrationspolitik wurde in einem Aufsatz in der Zeitschrift „Raumforschung und Raumordnung“ publiziert. Dort verwendete Zitatstrecken finden sich in gekürzter Form in diesem Beitrag.
}

ne empfindliche Störung bisheriger Handlungsroutinen“ gefasst, wobei der Prozess der Entscheidungsfindung unter Druck geschieht und durch Unsicherheit gekennzeichnet ist. Angenommen wird meist ein typischer Krisenverlauf, der sich in mehrere Eskalationsstufen untergliedert. Ausgehend von einem Plateau eines unterstellten „Normalzustandes“ folgt eine Phase von Auffälligkeiten, kleineren Störungen der Routine. Sie führen zu einer schwierigen und dann krisenhaften Situation. Auf dem Höhepunkt der Störung kommt es zu einer akuten Krise, die den Wendepunkt, die Klimax, einleitet und den Übergang in ein höheres Niveau eröffnet. In der Friedens- und Konfliktforschung dient dabei als quantifizierender Indikator für die Intensität des Konfliktes die mit den verschiedenen Eskalationsstufen verbundene Gewalthaftigkeit. Je nach dem Niveau der Eskalation werden erst verbale, dann körperliche Angriffe gezählt. Der Grad der Eskalation wird an der Zahl der Verletzten und anschließend an der Zahl der Toten festgemacht. In dieser Argumentationslogik handelt es sich angesichts der kontinuierlich hohen Zahl an Toten im Mittelmeer zweifelsfrei um eine Migrationskrise - so wird es auch in den von der EU selbst herausgegebenen Dokumenten formuliert (Hampshire, 2015). Allein in der ersten Hälfte des Jahres 2018 sind laut IOM (International Organisation for Migration) 1405 Menschen auf ihrer Flucht über das Mittelmeer umgekommen - Vermisste nicht eingerechnet. Entgegen den Erwartungen des UNHCR (United Nations High Commissioner for Refugees) steigt die Zahl wieder (Zeit online, 2018). Die europäische Migrationskrise, die wiederum nur ein Ausschnitt der globalen Migrationskrise ist, konzentriert sich in besonders zugespitzter Form auf Italien und führte zu einem dauerhaften Ausnahmezustand dort. Hier nun wurde der Ausnahmezustand zum „,neuen Normal“ der europäischen Grenzpolitiken (Castelli Gattinara, 2017:327). Wie bei Agamben (2002:27) beschrieben, wird der Ausnahmezustand zur Normalität und legitimiert die Existenz von Lagern, die ein Stück ,Land, das außerhalb der normalen Rechtsordnung gesetzt wird" sind. Das Lager konstituiert so neben den Ordnungsprinzipien Staat, Nation via Staatszugehörigkeit und Territorium eine eigene strukturierende Macht. Das Lager ist Ausdruck der Krise des Nationalstaatsprinzips (Hillmann, 2016:132). Es handelt sich vielfach um eine Kontrollkrise, d.h. vorhandene Regularien und Werkzeuge zur Beherrschung des status quo reichen nicht mehr aus und erfahren eine Ausweitung oder Neuauflage.

Typisch für fast alle Krisen ist, dass sie Anlass bieten, ein hohes $\mathrm{Ma}$ an Wissensproduktion zu generieren und Experten auf den Plan zu rufen. Auf diese Weise wird die Krise nach und nach zu einem machtgeladenen Narrativ, das wiederum selbst ein Werkzeug zur Erzeugung neuen Wissens über sozialräumliche Transformationen wird und politische Entscheidungen beeinflusst (Rigo, 2018:507). Im Falle der Migrationskrise ist typisch, dass die MigrantInnen selbst, die als Auslöser der Krise gesehen werden, kaum angehört werden. Krisen sind damit Ausnahmesituationen, die vor allem 
auch den Medien neue Interpretationen auf eine schon länger bestehende Problemlage ermöglichen. Im Falle der Migrationskrise bedienen sie sowohl in der Regenbogenpresse als auch in den seriösen Magazinen immer wiederkehrende Stereotypen: Sicherheit, Kriminalität und Viktimisierung, außerdem Konkurrenz auf dem Arbeitsmarkt (Greussing and Boomgaarden, 2017:1763). Die Berichterstattung über die Flüchtlingskrise trug in Italien dazu bei, dass sich die bereits vorhandenen Ressentiments gegenüber Flüchtlingen aus Iran und Irak verschärften. So ist die Angst vor den Auswirkungen der Zuwanderung auf die wirtschaftliche Krise in der Bevölkerung laut einer Studie der Pew-Foundation größer als die Angst vor Terrorismus und Unsicherheit. Die Rechtsaußen-Parteien Lega Nord, Fratelli D'Italia sowie das M5S (movimento cinque stelle) gewannen die lokalen und nationalen Wahlen, und die rechtsradikalen Parteien Forza Nuova und die neofaschistische CasaPound Italia profitierten von einer panikheischenden Berichterstattung (Castelli Gattinara, 2017:325). Die Migrationskrise wurde als Legitimationskrise des italienischen Staates innerhalb des Mehrebenensystems der EU wahrgenommen. Diese GovernanceKrise übersetzte sich in eine Vertrauenskrise (Carmagnani and Pastore, 2018). Während sich die europäischen Bürger mit großer Selbstverständlichkeit einer so großen Mobilität wie nie zuvor in der Geschichte erfreuen, sei es durch Tourismus oder durch die innereuropäische Migration, wird von vielen Bürgern zugleich erwartet, dass die Mobilität von Drittstaatlern stark eingeschränkt oder sogar unterbunden wird.

In dieser Migrationskrise treffen, so wird im Folgenden gezeigt, unterschiedliche Krisen mit ihren unterschiedlichen Zeitlichkeiten aufeinander: (a) eine Vulnerabilitätskrise in den Herkunftsregionen, die ursächlich für die individuelle Krise des Migranten und der Migrantin und deren/dessen Handlungslogiken ist, (b) eine Krise der nationalstaatlichen Regulierung von Migration und (c) eine Krise der Städte im Umgang mit Migration. Die Krise, so wird hier gezeigt, ist nur als eine Mehr-Ebenen-Assemblage überhaupt zu verstehen. In dieser Mehr-Ebenen-Assemblage wird den Akteuren eine bestimmte Rolle zugewiesen, sie verfügen aber dennoch über Handlungsspielräume und sind in der Lage, durch den Handlungsdruck soziale Innovationen zu entwickeln.

\subsection{Die Vulnerabilitätskrise in den Herkunftsländern}

Schon seit den 1980er Jahren entwickelte die Sozialgeographie Ansätze und Modelle der Vulnerabilitätsforschung. Ursprünglich entstanden diese um zu verstehen, wie Hunger und Armut besser bekämpft werden können. Denn es war weniger das Fehlen von Nahrungsmitteln, sondern der nicht vorhandene Zugang zu Nahrungsmitteln, der sich als Hauptursache für die Verwundbarkeit der Bevölkerung vor Ort herausstellte (Sen, 1981). Auf dieser Erkenntnis aufbauend, interpretierten Watts und Bohle (1993) die spezifische Verwundbarkeit von Individuen und Gruppen als verortet in einem Netz von sozialen Beziehungen, insbesondere von Machtbeziehungen und Verfügungsrechten. Der strukturelle Zugang der Autoren zur Thematik führte weg von der intensiven Beschäftigung mit den Naturereignissen, d.h. den Dürren oder Überschwemmungen an sich. Ihr Blickwinkel rückte stattdessen die Coping-Strategien und die Agency der vulnerablen Gruppen in den Mittelpunkt der Analyse. Ähnlich wie in den Modellen der eskalierenden Krisenverläufe, gingen die Autoren von einer zunehmenden Krisenempfindlichkeit aus, die sich durch eine Verkettung von katastrophenauslösenden Ereignissen (concatenations) und gegenläufigen Tendenzen der Widerstandsfähigkeit beschleunigen konnten. Das Niveau der Verwundbarkeit erhöhte sich jeweils nach der überwundenen Krise für die unterschiedlichen gesellschaftlichen Gruppen in unterschiedlichem Maße und war bedingt durch die capabilities der Akteure, nicht durch ein ,lack of want" (Chambers, 1989). In ihren Arbeiten identifizierten die beiden sozialgeographischen Autoren „Migration“ dezidiert als Coping-Strategie in gesellschaftlichen Krisensituationen. Abwanderung erfolgte jeweils nach einer Phase der Verarmung und stand in ihren Modellen zeitlich unmittelbar vor der „Auszehrung durch Krankheit und Tod“. Migration in die Zentren stellte sich für sie als eine der wichtigsten Ausweichstrategien dar und zwar nachdem der Haushalt eine Phase der Anpassung der Familiengröße, eine Intensivierung der Landwirtschaft und die Ausnutzung aller vorhandenen ökonomischen Ausweichstrategien bereits durchlaufen hatte.

Es spricht viel dafür, die steigenden Zahlen der Binnenmigration auf dem afrikanischen Kontinent, insbesondere in den Sub-Sahara-Ländern, mit einer durch den Umweltwandel weiter erhöhten Vulnerabilität der Bevölkerung zu begründen. Länder wie Ghana sind mittlerweile durch veränderte Regenfallmuster und zunehmende Dürren gekennzeichnet, was nachweislich einen Rückgang der Fertilität in der Bevölkerung und mehr Abwanderung mit sich bringt (Adaaven, 2015:212f.). Studien über den Zusammenhang von Umweltwandel und Migration betonen zwar die Multikausalität von Migrationsentscheidungen, sie zeigen aber auch, wie Migration zu einem selbstverständlichen Teil der lokalen Kultur wird und seit vielen Jahren vermehrt als Strategie der Risikominimierung dient (van der Geest, 2011; Hillmann and Ziegelmayer, 2016). Auch Haushaltsmitglieder, die kein erhöhtes Vulnerabilitätsrisiko haben, entscheiden sich für eine Abwanderung und ziehen weitere Angehörige nach (Jarawura and Smith, 2015:262). Darüber hinaus sind in Teilen des sub-saharischen Westafrikas grenzüberschreitende Migrationen die Normalität, nicht die Ausnahme. Entsprechend machen interne und interregionale Migrationen, vor allem in die Hauptstädte, den allergrößten Teil des afrikanischen Migrationsgeschehens aus. Die internationalen Migrationsrouten, wie zum Beispiel von Ghana über Niger und Libyen nach Europa, folgen jahrhundertealten Handelsrouten (Lucht, 2017). In den Transitländern Nordafrikas sorgen Migrationsindustrien dafür, dass der Nachschub an MigrantInnen gesichert ist (Gammeltoft-Hansen and Nyberg 
Sørensen, 2013). So existieren in Agadez ganze Siedlungen, in denen MigrantInnen darauf warten, ihren Weg nach Europa (wieder) zu versuchen. Migration von Afrika nach Europa ist ein großes Geschäft geworden, bei dem Kriegstreiber wie der IS involviert sind und hier dankbar das „Management“ übernahmen (Greenhill, 2016:329).

Wer an den Küsten Italiens anlandet, ist mit hoher Wahrscheinlichkeit schon Monate und Jahre unterwegs und hat Phasen der Immobilität durchlebt, weil die finanziellen Ressourcen verbraucht waren oder weil er/sie krank wurde oder keine Arbeit fand. Im Vorfeld und während ihrer Reise nach Europa mussten Schleuser bezahlt und Grenzposten bestochen werden. In den Transitländern findet sich dann keine Arbeit, hohe Mieten müssen bezahlt werden. Die meisten MigrantInnen verfügen - anders als in der Forschungsliteratur immer wieder beschrieben - eben nicht über die sozialen Unterstützungsnetzwerke. „Bleiben“ ist für sie indes nur die zweitbeste Option (Schapendonk, 2008:135), Zurückgehen ist auch keine Lösung (Lucht, 2017). Die „gescheiterten" MigrantInnen verleugnen sich der Familie gegenüber. Bei der Überfahrt umzukommen ist unter Umständen besser als mit leeren Händen in das stagnierende Land zurückzukehren und die Schmach der Verliererin auszuhalten (Alpes, 2019). Bei vielen sind der Traum und die Hoffnung auf ein anderes Leben in Europa so stark, dass das Migrationsprojekt zum alleinigen Lebensinhalt wird. Auch wenn es bei der ersten und zweiten Überfahrt nicht gelingt, folgen weitere Versuche. Gerade wenn man schon viel investiert hat und von Seiten der Angehörigen große Erwartungen bestehen, besteht ein enormer Erfolgsdruck. In Ländern wie Ghana hat sich überdies die nationale Erzählung über Migration als Lösung bestehender Probleme verfestigt (Manuh, 2006:24f.; Awumbila et al., 2016). Wie stark diese Vorstellungen über das Ziel Europa in der Gedankenwelt der westafrikanischen MigrantInnen verankert sind, zeigt sich in verschiedenen qualitativen Untersuchungen zu deren trajectories, den kollektiven raumzeitlichen Gefügen der Migration. Der Fokus der Forschung richtet sich dann auf die Agency der MigrantInnen (Kleist and Thorsen, 2017; Hillmann et al., 2019). Die Forscher sprechen von einem „Mobility paradox“ und meinen damit, dass einerseits eine nie gekannte Menge an Bildern der Hoffnung zirkuliert, die Migrationsanreize setzt und andererseits immer mehr Menschen von sicheren Migrationsrouten ausgeschlossen sind bzw. keine legalen Zugänge nach Europa mehr bestehen. Der Zugang zu sicheren Migrationswegen wird so zu einem der entscheidenden Kriterien von Ungleichheit (Kleist and Thorsen, 2017). Für viele MigrantInnen endet die Hoffnung in der libyschen Wüste - es sterben ebenso viele dort wie auf der Überfahrt (Lucht, 2017; Plambech, 2017; Klepp, 2011:174ff.).

Die erhöhte Vulnerabilität großer Bevölkerungsschichten in den Herkunftsregionen wird damit zu einem Bestandteil der Migrationskrise. Diese strukturelle Krise übersetzt sich in eine Krise der Individuen, denn diese suchen eine reelle, d.h. zeitlich umsetzbare Lösung für ihre existenziellen Pro- bleme. Je größer die Krise vor Ort ist, desto stärker versuchen die Individuen ihre Handlungslogiken nach außen auszurichten. Migration ist unter Umständen die einzige Möglichkeit, der wahrgenommenen Perspektivlosigkeit des Herkunftslandes innerhalb der eigenen Lebenszeit zu entfliehen, weil eine persönliche Entwicklung im Land nicht möglich ist. Ihre Wanderungen sind, einmal in Europa angekommen, zirkulär und fragmentiert sowie durch Sekundärmigrationen geprägt (Fontanari, 2016; Borri, 2016). Mehr Mobilität wird zu einer Option der Risikominimierung.

\subsection{Die italienisch-europäische Migrationskrise}

Die Krise polarisiert und definiert die Linie zwischen Wirtschaftsmigration und Flucht neu, dient der Neuverhandlung der Balance zwischen Aufenthaltsländern und Ankunftsländern der Migration. Wissenschaftler bemängeln, dass die Krisenterminologie EU-zentristisch ist, weil sie unbeachtet lässt, dass es konstante Migration in vielen Teilen der Welt gibt. Sie suggeriert, dass es eine zeitliche Beschränkung dieses Zustandes der Zuwanderung gibt und zugleich, dass die Prekarität der anlandenden Menschen die Möglichkeiten einer humanitären Antwort unterläuft. Einmal in Europa angekommen, treffen die MigrantInnen dort auf die Krise des italienischen Nationalstaates (Fratesi and Percoco, 2014; Caneva, 2014), die an die humanitäre Krise direkt gekoppelt ist.

Wie in den anderen europäischen Ländern auch, reagiert Italien auf die Krise im Rahmen von multi-level governance, in der zunehmend Städtenetzwerke und subnationale Foren Einfluss auf Integrationsprojekte und temporäre Schutzmaßnahmen nehmen und durch diese Europäisierung AntiMigrationsressentiments auf lokaler Ebene zu umgehen suchen (Panizzon and van Riemsdijk, 2018:6f.). Im Gegensatz zu anderen europäischen Ländern hat Italien erst nach dem Berliner Mauerfall überhaupt angefangen, seine Asylpolitik zu strukturieren. Auslöser war seinerzeit die Angst vor einer unkontrollierten Zuwanderung aus dem zerfallenden Jugoslawien und Albanien (Campesi, 2018). Seitdem entwickelte sich das italienische Asylsystem entlang zweier höchst unterschiedlicher Aufnahmemodi. Ab 1995 (Dekret N. 451/1995) entstanden einerseits Aufnahmezentren, die direkt durch das Innenministerium geleitet wurden und die vor allem in Süditalien in Nähe der Anlandungsorte positioniert waren. Es handelte sich um große Aufnahmeeinrichtungen (Centri di primo soccorso e assistenza), die 2002 zu den Erstaufnahmezentren für Asylsuchende umfunktioniert wurden (Centri di identificazione, Gesetz 189/2002) und dann wiederum zu Centri di Accoglienza per richiedenti asilo (Gesetz 25/2008). Daneben entstanden auf Initiative verschiedener NGOs in Kooperation mit den lokalen Behörden und Verwaltungen kleinere Aufnahmezentren, die seit 1999 in einem Projekt namens Azione Commune ein erstes Netzwerk für Asylbewerber und Geflüchtete aus dem Kosovo etablierten. Dieses Netzwerk wurde 2001 in das nationale Asylprogramm (Programma nazionale asilo) überführt und durch 
ein Abkommen zwischen dem Innenministerium, den italienischen Kommunen und dem UN-Hilfswerk UNHCR (United Nations High Commissioner for Refugees) formalisiert (Campesi, 2018:492). Diese Zweiteilung folgte der Logik, dass die Erstaufnahme der Flüchtlinge besser durch Sammelunterkünfte gelöst werden könnte, während die NGObetriebenen Aufnahmezentren vielmehr auf die langfristige Eingliederung der MigrantInnen eingehen sollten. Das italienische Asylsystem geriet unter Druck, als die Libyen-Krise 2011 Massenwanderungen auslöste. Im Zuge dieser humanitären Krise wurde dann eine Reihe neuer Aufnahmezentren eröffnet. Sie wurden zunächst der Protezione civile (Zivilschutz), dann den Präfekturen, d.h. den jeweiligen Regionalbüros der Zentralregierung, unterstellt. Im Jahr 2015 wurde das italienische Asylsystem reformiert. Es besteht seitdem aus drei Segmenten, denen jeweils unterschiedliche Philosophien zugrunde liegen. Erstens existieren die durch die EU eingeforderten Hotspots, in denen auch die Identifizierung stattfindet, wo Fingerabdrücke abgenommen werden und die auf eine Sicherheitsaufbewahrung zielen (sie gründen sich auf das Dekret 451/1995 als Rechtsgrundlage). Zweitens finden sich spezialisierte Erstaufnahmezentren zur Aufnahme von Flüchtlingen (Centri di prima accoglienza, begründet durch Artikel 9 und 10 des Dekrets 142/2015). Sie folgen einer Beschränkungslogik. Drittens besteht ein Zugang zu den Aufnahmezentren durch das SPRAR-System oder aber - in Ausnahmefällen - zu den außerplanmäßigen Aufnahmezentren. Hier folgt die Aufnahme der MigrantInnen einer Zerstreuungslogik. Zum Höhepunkt der Migrationskrise wurden den Hotspots ${ }^{4}$ bis zu 8000 Flüchtlinge zugewiesen und schließlich nach dem Schlüssel 3 zu 1000 Einwohner durch ANCI (Associazione Nazionale Comuni Italiani) auf die Regionen verteilt. Die Zahl der unbegleiteten minderjährigen Flüchtlinge stieg auf 18486 Menschen (7/2017, Ministero Dell'Interno, 2017). Ein Grundproblem dieser Art dieses nach und nach eingeführten Grenzmanagements besteht darin, dass der Diskurs über die humanitäre Hilfe und die entsprechenden Praktiken zu einer symbolischen Unterordnung der MigrantInnen führte. Im Fokus der Aufmerksamkeit stand nun immer weniger das Recht auf Menschenwürde, sondern die Gnade, den Bedürftigen Aufenthalt zu gewähren (Cuttita, 2018).

Mit den Zuspitzungen der verschiedenen Krisen kam es zu einer immer stärkeren Entkopplung der lokalen Politiken von den nationalen Integrationspolitiken. Die ,,außerplanmäBigen" und temporär angelegten Zentren wurden zu einer tragenden Säule des Systems insgesamt (Campesi, 2018:494). Schon die nationalen Immigrationspolitiken zwischen 2007 und 2017 hatten sich im Kontext mehrerer Krisen entwickelt: der 2008 beginnenden Finanzkrise und der durch den arabischen Frühling 2011 und durch den Krieg in Libyen ausgelösten Flüchtlingskrise. Die 2016 ausgerufene Emer-

\footnotetext{
${ }^{4}$ Es existieren vier Hotspots: Lampedusa, Pozzallo, Trapani und Taranto.
}

genza Nord stellte somit lediglich einen Höhepunkt einer in der öffentlichen Diskussion breit diskutierten Notfallsituation dar (Caponio and Cappiali, 2018:115ff.; Campomori and Caponio, 2017). Der Höhepunkt der Migrationskrise setzte 2014 ein, als rund 170000 Menschen anlandeten, 2015 waren es 154000 und 2016 181 436. 2017 sank die Zahl auf rund 119000 Menschen. Damit war Italien im Jahr 2017 mit rund 70 Prozent aller Anlandungen in Europa immer noch das wichtigste Ankunftsland im Mittelmeerraum. Es kamen vor allem Menschen aus Tunesien, Eritrea, Nigeria, Sudan und der Elfenbeinküste.

Italien hatte in den Jahrzehnten zuvor zwei Massenlegalisierungen durchgeführt (Gesetz Turco-Napolitano, 1998 und Gesetz Bossi-Fini, 2002), die den Aufenthaltsstatus von 870000 Menschen legalisierten. Sie lösten so eine über Jahre hinweg schwierige Situation massenhaft illegaler Aufenthalte im Land auf. In Italien existierte über Jahre so etwas wie eine ,starke Lobby der Schwachen“, die zur Akzeptanz der krisenhaften Situation führte (Zincone, 2011). Es war eine zufällige Interessensallianz heterogener Akteure: zwischen den kleinen und mittleren Unternehmen, die billige Arbeitskräfte brauchten, und den katholisch geprägten NGOs, die sowohl den humanitären Aspekt der Einwanderung betonten als auch Migrantinnen in den Haushalts- und Pflegearbeitssektor vermittelten. Drittens setzten sich die Gewerkschaften für die Inklusion der MigrantInnen in soziale und ökonomische Rechte ein und machten sich für eine Regularisierung der ausländischen Arbeiternehmer, zum Schutze der einheimischen Bevölkerung vor Dumpinglöhnen, stark. Dementsprechend zielten die weiteren Legalisierungen (2009) auf den Pflegesektor bzw. sie ermöglichten Legalisierungen in allen Arbeitsmarktsegmenten (2012), stellten jedoch hohe Anforderungen an die Arbeitgeber. Dieser internen Kontrolle durch Legalisierungen stellten die italienischen Behörden mehrere Maßnahmen der externen Grenzkontrollen mit finanzieller europäischer Unterstützung an die Seite (die Operation Mare Nostrum, 2013; Triton, 2014; EU Navfor Med Sophia, 2015), die schließlich immer stärker der Bekämpfung der Menschenhandelskriminalität dienten.

Im April 2015 ertranken 700 MigrantInnen bei einer Seenotrettung, was sowohl eine humanitäre als auch eine Kontrollkrise nach sich zog. MigrantInnen ohne gültige Aufenthaltspapiere und Asylbewerber wurden nun stärker in den Blick genommen. Gemäß der Dublin-Konventionen (Dublin I, 1990, Dublin II, 2003, Dublin III, 2013) sah die „European Agenda on Migration" (COM2015/240), die von der EU-Kommission im Mai 2015 angenommen wurde, auch in Italien die Einrichtung von Hotspots vor. In diesen Hotspots sollte erste Hilfe geleistet und die Identifikation der Ankommenden durch Fingerabdrücke u.v.m. dokumentiert werden. Um zu ermitteln, welche MigrantInnen einen Flüchtlingsstatus beantragen konnten, arbeiteten Mitarbeiter von Frontex, Easo (European Asylum Support Office), Europol (European Police Office) und Eurojust (EU Judicial Cooperation Unit) mit der italienischen Polizei zusammen. Am 12. April 
2017 wurden schließlich durch das Minniti-Orlando-Dekret neue, restriktivere Kontrollmechanismen eingeführt (Decree no. 13, in 2017). Das Dekret weitete die Rolle der CIE (Centres of Identification and Expulsion - Centri di Identificazione e Espulsione), jetzt CPR genannt (Centres of Permanence and Repatriation - Centri di Permanenza e Rimpatrio), aus. Ziel war die Rückführung derer ohne Rechtsanspruch auf internationalen Schutz. Ab jetzt konnten sich die Asylbewerber nicht mehr wie zuvor an die normalen Gerichte wenden, sollte ihr Asylbegehren abgelehnt werden.

Seitdem spielte die humanitäre und die ökonomische Krise die wichtigste Rolle für die Zusammensetzung der Zuwanderungsbevölkerung (Caponio and Cappiali, 2018:121f.). Die Kombination einer Reduzierung der Zulassungsquoten und ein schwächelnder italienischer Arbeitsmarkt leitete mittlerweile einen Rückgang der illegalen Migration ein. Die Statistiken über die illegalen Grenzübertritte belegen einen deutlichen Rückgang (von 24528 im Jahr 2004 auf 7713 im Jahr 2013). Dieser faktische Rückgang der Zuwanderung wird in Presse und Öffentlichkeit indes kaum wahrgenommen. Von der Öffentlichkeit weitgehend unbemerkt, hatten AsylbewerberInnen nach und nach die ArbeitsimmigrantInnen ersetzt. Die zeitliche Verstetigung durch Zwischenlösungen wurde zum Merkmal der italienischen Migrationspolitiken. Festhalten lässt sich insgesamt, dass die Einwanderung nach Italien weitgehend über die Arbeitsmarktbedarfe und zufällige Ereignisse, nicht aber durch gezielte Einwanderungspolitiken gesteuert wurde.

\section{Die Krise der Städte durch Migration}

Städte sind die Orte, wo Migration gelebt und bearbeitet wird. Es sind die MigrantInnen, die durch ihr Agieren im Raum versuchen, ihre Handlungsspielräume zu erweitern und durch ihre Kontakte zu vorhandenen Netzwerken transnationale Kontakte für sich zu nutzen (Pries, 2007; Borri, 2016). Sie navigieren auf Grundlage ihres Wissens durch den städtischen Raum und kommen zunächst bevorzugt in bereits migrantisch geprägten Nachbarschaften unter. Städte wie Rom, in denen Tausende MigrantInnen 2017/2018 in verlassenen Gebäuden lebten, gerieten unter Druck. Die Räumung von 800 Eritreern und Äthiopiern unter Einsatz von Wasserwerfern im August 2017 in der Via Curtatone und $\mathrm{Ci}$ necittà können als Beispiele für diese bestehende Überforderung der Ankunftsstädte, die sowieso schon mit Problemen aller Art kämpften, gelten. Die Migrationskrise stellte insofern eine Verlängerung der über viele Jahre hinweg angelegten Krise des Sozialstaates in den Städten dar, weil sie zu einem bereits angespannten Arbeits- und Wohnungsmarkt hinzutrat.

Da die MigrantInnen in den Städten selbst keine Arbeit mehr fanden und die Arbeit in der Landwirtschaft und auf den Schlachthöfen prekär und schlecht bezahlt war (wenn überhaupt), wanderten sie von Italien aus weiter, z.B. nach
Frankreich, Großbritannien und Deutschland (Ambrosini, 2018:65). Ein pragmatisch agierender und informell organisierter Arbeitsmarkt mit wenig formalen Rekrutierungswegen und durch intermediäre Akteure gesteuert, zog weiter ImmigrantInnen an. Oft handelte es sich um leicht auszubeutende, da rechtlich nicht geschützte, abgelehnte Ayslbewerber (Finotelli and Echeverría, 2017), die zum Beispiel im Caporalato-System in der Landwirtschaft schufteten. Das Problem lag im ständigen Notfallmodus, wie es Alexandra Benvenuti vom Aufnahmeheim in Via Caffarò in Genua zusammenfasst:

In Italien arbeitet man immer im Notfallmodus, seit Jahren reagiert man so strukturell auf die Migrationsströme. Die Leute sind in unseren Zentren zu Gast und sie warten darauf, den Aufenthaltsstatus als Flüchtling zu erhalten. Wenn die Antwort negativ ist, verlieren sie das Recht, sich in diesen Strukturen aufzuhalten. Das führt dann dazu, dass diese informellen Siedlungen entstehen und die Nicht-Integration einsetzt. (zit. nach Giordanella, 2016)

Der in der Literatur beschriebene "turn to the local“ (Asselin et al., 2006; Glick Schiller and Caglar, 2011), gemäß dem sich die Stadtpolitiken zunehmend abgekoppelt haben von den nationalen Politiken (s.o.), eben weil sie die Möglichkeit zur horizontalen Vernetzung mit MigrantInnenorganisationen und NGOs haben, wie auch vertikal mit globalen Institutionen kooperieren können, ist damit ein Zeichen der Krise des Nationalstaates.

Die Krisengovernance der Städte, hier definiert als unmittelbares, spontanes, vorbehaltloses und wohltätiges Agieren in einer Ausnahmesituation außerhalb demokratisch legitimierter Prozesse, vollzieht sich auf mehreren Handlungsebenen zugleich (Panizzon and van Riemsdijk, 2018:5, nach einer Definition von Streeck). Italien hatte diesbezüglich eine ganz eigene lokale Governance aufgebaut, das SPRARSystem, das im Folgenden in der gebotenen Kürze vorgestellt wird.

\section{SPRAR - ein vorzeitig aufgehobenes Experiment}

Das System zur Aufnahme von Flüchtlingen und MigrantInnen stellte sich von Anbeginn an als rechtlich fragmentiert, stark bürokratisiert und uneinheitlich verwaltet dar (Klepp, 2015). Wenn einE MigrantIn an den italienischen Küsten anlandete, kam er oder sie zunächst in den Hotspot. Wie oben beschrieben, verästelte sich das weitere Unterbringungssystem dann - wobei Theorie und Praxis weit auseinanderklafften. Lag kein Asylantrag vor, wurden die MigrantInnen in das System CIE (Centro di identificazione e espulsione)/CPR ${ }^{5}$ vermittelt, wo sie sich 90 Tage bzw. maximal

\footnotetext{
${ }^{5}$ Es gibt vier dieser Zentren: Torino, Roma, Brindisi und Caltanissetta.
} 
12 Monate aufhalten sollten bzw. konnten. Wurde von dem oder der MigrantIn Asyl beantragt, erfolgte eine Überführung in ein CAS (Centro di Accoglienza Straordinaria) oder in ein CPA (Centro di prima accoglienza), wo die Identität festgestellt und der Antrag auf Asyl gestellt wurde. Nach einem Aufenthalt dort konnte der oder die MigrantIn in das SPRAR-System (Sistema di protezione per richiedenti asilo e rifugiati) eingegliedert werden, das eine vollständige Integration beabsichtigte und das weit über Unterkunft und Verpflegung hinausreichen sollte. Das SPRAR ${ }^{6}$-System existierte seit 1999 und ging ursprünglich aus Bottom-up-Ansätzen der Stadtentwicklungspraxis hervor. Strukturiert war es in einer Mehrebenenlogik. National finanziert, zielte es auf eine Vernetzung der lokalen Ämter und kooperierte eng mit dem Dritten Sektor, d.h. den Non-Profit-Organisationen. Unterstützt wurden insbesondere lokale Netze, die alle AkteurInnen an einen Tisch brachten. Im März 2018 gab es in ganz Italien 876 aktive Projekte, 35869 Nutznießer und über 1200 involvierte Kommunen. Diese Kommunen waren größtenteils in Süditalien angesiedelt; viele Kommunen in Norditalien verweigerten sich und protestierten auch gegen die Ansiedlung von CAS. Die Formen des Widerstands reichten von Slogans wie „Herr im eigenen Haus“ bis zu konkreten Sabotage-Aktionen, indem den Unterkünften der Strom abgedreht wurde oder sich ein dauerhafter Protestreigen vor der Asylbewerberunterkunft aufbaute (Ambrosini, 2018).

Interessant ist, dass das SPRAR-System auf das Einverständnis und die Unterstützung der Kommunen angewiesen war. Trotz der finanziellen Unterstützung lehnten viele Kommunen eine Beteiligung am System ab und so gab es eine zu geringe Zahl an Plätzen. Durch das im April 2017 beschlossene Minniti-Orlando-Gesetz sollten die Erstankömmlinge ja innerhalb von 48 Stunden weitervermittelt werden (in einem centro di accoglienza oder einem hub regionale). In der Praxis wurden die Zeiten meist nicht eingehalten, weil zu wenige Kommunen Plätze zur Verfügung stellten. So kam es, dass in der Praxis unterschiedliche Unterbringungsmodi nebeneinanderher bestanden (CAS, wenn es keine Einwilligung für ein SPRAR gab) und sich seit vielen Jahren eine widersprüchliche, in höchstem Maße undurchsichtige Situation entwickelt hat. Es liegt keine Übersicht über die finanzielle Situation der einzelnen Zentren vor, auch nicht darüber wie und wofür diese die Mittel des Innenministeriums verwenden (Klepp, 2015:179). Bei Prüfungen zeigte sich, dass einige Zentren lediglich auf dem Papier existierten und dass Fördergelder, durch italienische und europäische Institutionen investiert, einfach verschwanden. Auch die Abläufe in den Zentren waren unklar, präzise Zahlen fehlten gänzlich, gemeinsame Strategien gab es nicht. Die Präfekten, die über die Zuteilung der MigrantInnen in die Zentren zu entscheiden hatten, besaßen in dieser Situation die größte Entscheidungsmacht. Wie prekär die Governance der Migration in Italien

\footnotetext{
${ }^{6}$ Das SPRAR-System (Sistema di protezione per richiedenti asilo e rifugiati).
}

seit langem war, zeigte sich unter anderem 2013 als mehrere Aufnahmezentren aufgrund miserabler Zustände (teilweise durch die Intervention der EU) geschlossen werden mussten. Eine Sonderlösung wurde gefunden. Die Flüchtlinge erhielten von den italienischen Behörden eine Zuwendung von 500 Euro und ein Visum, das es Ihnen erlaubte, sich frei durch das EU-Territorium zu bewegen, bevorzugt nach Deutschland weiterzureisen (Benigni and Pierdicca, 2016:5; Borri, 2016; Fontanari, 2016) - wozu diese von den Behörden dann auch aufgefordert wurden. Die Kommunen waren direkt in das SPRAR-System eingebunden, und sie haben diese Chance zum Beispiel dazu genutzt, um sich um die vielen unbegleiteten Minderjährigen zu kümmern. Wie uns die zuständige Sozialdezernentin von Genua erklärte:

Innerhalb des SPRAR-Systems gibt es eine Vereinigung, die sich um die Minderjährigen kümmert und das heißt, dass ich, wenn ich etwas für die unbegleiteten Minderjährigen tun will, dann eröffne ich was für die Jugendlichen, die direkt von den Schiffen kommen. Wir haben aber nicht genug Plätze, daher werden die Jugendlichen dann oft in den Unterkünften für die Erwachsenen mit untergebracht.

Im Oktober 2018 hat die italienische Regierung unter Innenminister Salvini die Flüchtlinge von einer Unterbringung im SPRAR-System ausgeschlossen und lässt diese nur noch in den CAS unterbringen. In diesen Zentren wurde zudem eine Reihe von Angeboten (psychologische und medizinische Versorgung, Sprachunterricht, Vorbereitung auf den Arbeitsmarkt) gekippt und die Tagesrate von 35 auf 20 Euro gekappt (s.u.). Begründet wurde dieser Schritt damit, dass die meisten Asylanträge unter der neuen Regierung sowieso abgelehnt würden und dass es daher sinnlos sei, überhaupt Geld in Italienischkurse zu investieren oder die Menschen in den italienischen Arbeitsmarkt zu integrieren.

\section{Fallbeispiel Genua}

\subsection{Bedeutung von und Umgang mit Migration in der Stadt Genua}

Genua gehörte zusammen mit den Städten Mailand und Turin zu den industriellen Zentren Italiens - was immer ein Hinweis auf eine starke Einwanderungsgeschichte ist. Zunächst hatte sich die Stadt mit der Industrialisierung als wichtiger Auswandererhafen nach Übersee etabliert und die Zuwanderung aus den ländlichen Räumen und den Bergen im Hinterland in die Stadt hinein zur Arbeit in den Fabriken aufgesogen. Während der Nachkriegszeit transformierte sich die Stadt schnell. Mit dem Wirtschaftsboom in den 1960er Jahren kamen dann die Zuwanderer aus Süditalien und von den Inseln zur Arbeit in der Schwerindustrie und im Hafen. Seit Mitte der 1980er Jahre erlebte Genua schließlich eine Zuwanderung aus den Ländern des globalen Südens, vor allem 
aus Nordafrika (überwiegend männliche Migranten) und aus Equador (überwiegend Migrantinnen, die im Pflegesektor arbeiteten). 1986 belief sich die Zahl der Ausländer noch auf lediglich 5222 Personen und stellte damit lediglich ein Zehntel der heutigen ausländischen Bevölkerung dar. Seit 2011 hat sich dieser Bevölkerungsanteil zwischen 50 000-55000 Personen eingependelt, wobei seit 2014 die Zahlen sogar leicht zurückgehen. Daten über die Zahl der Menschen, die sich ohne gültige Aufenthaltsgenehmigung in der Stadt aufhalten, gibt es nicht. Doch man kann annehmen, dass es eine gewisse Zahl an Visa-Overstayern, d.h. Menschen mit abgelaufenen Aufenthaltsgenehmigungen, gibt. Doch auch diese Situationen, so geht aus den Interviews hervor, sind in der Tendenz rückläufig.

In Genua richteten sich die migrationsbezogenen Aktivitäten der Stadtverwaltung - ähnlich wie auf nationaler Ebene auf die Bewältigung von akuten Krisensituationen (insbesondere Flüchtlingsunterbringung) und die Integration von Familien im Rahmen der allgemeinen Sozialpolitiken, in denen die Zuwanderer versorgt wurden wie alle anderen Stadtbürger auch. Ein eigenständiges und koordiniertes städtisches Integrationsprogramm existierte nicht, jedoch gab es punktuelle Maßnahmen von Seiten der verschiedenen Ressorts der Stadtverwaltung (Hillmann and Calbet i Elias, 2019). Die städtischen Behörden arbeiteten zwar nicht immer zusammen, doch telefonierte man in Notsituationen miteinander, um eine pragmatische Lösung zu finden. Man suchte aktiv gemeinsame Lösungen für akute Problemlagen. Die ehemalige Sozialdezernentin fasst dies so zusammen:

Wir arbeiten viel zusammen. Die Idee, die wir haben, ist, dass wir eine Koordination für eine gelingende Aufnahme finden. Etwas, bei dem sich alle engagieren, um ein bisschen mehr zu machen als nötig. Es ist eine Menge Energie im Umlauf und das muss nicht unbedingt von der Kommune koordiniert werden.

Einmal abgesehen von dieser städtischen Integrationspolitik ,unter dem Radar“ wurde auf die Flüchtlinge ad hoc und durch den ,terzo settore“ uneinheitlich reagiert. Sie wurden über das SPRAR-System in der Stadt verteilt und durch das Innenministerium finanziert. Für den Unterhalt gab es einen Zuschuss von 40 Euro am Tag, wovon die Kosten für die Unterbringung und medizinische Versorgung abgezogen wurden (12,80 Euro), die Trägerstruktur (16,20 Euro) und deren Instandhaltung subventioniert (7,80 Euro) als auch Integrationskurse und psychologische Hilfe finanziert (3,20 Euro). Zwischen 2,00 und 2,50 Euro waren als Taschengeld zur freien Verfügung für den Asylbewerber vorgesehen (Giordanella, 2016). Im öffentlichen Diskurs wurden vornehmlich die damit verbundenen Kosten thematisiert und die Krisensituation hervorgehoben. Einer der führenden Migrationsexperten betont, dass eine solche Sichtweise stark verkürzt ist und zudem die Problematiken nur verschiebt:
Das mit den Kosten stimmt, doch das meiste, was wir in die Flüchtlinge finanziell investieren, bleibt auch bei uns, unmittelbar vor Ort, du stellst Leute ein, kaufst Essen. Das übersehen die meisten. [...] Das Problem ist, dass die Menschen deren Asylgesuch abgelehnt wird, nicht weggehen und dann aus den Unterkünften raus müssen. Sie bleiben bei Bekannten, und so entstehen Situationen, die wirklich problematisch und sichtbar sind.

In der Altstadt von Genua, dem Centro Storico, und in Sampierdarena, etwa $4 \mathrm{~km}$ vom Zentrum entfernt, sind Gebiete entstanden, in denen sich ein hoher Anteil an eingewanderter Bevölkerung findet. Diese Nachbarschaften sind durch Beschaffungskriminalität und prekäre Situationen gekennzeichnet. Via Prè im Zentrum der Altstadt ist inzwischen fast überwiegend von afrikanisch-stämmigen MigrantInnen bewohnt und voller Geschäfte, die von den MigrantInnen betrieben werden. Eine der BewohnerInnen, die sich in einer Nachbarschaftsinitiative engagiert, fühlt sich von den Ordnungskräften und den Institutionen allein gelassen. Sie sieht die MigrantInnen als leichte Beute für mafiöse Aktivitäten:

Letzte Woche sind hier um die Ecke 23 Drogendealer festgenommen worden, das waren aber alles nur die Läufer, alles Asylbewerber, die werden für die Dienste genutzt. Der Pool, aus dem die fischen können, ist so unermesslich groß.

In der Altstadt überlagern sich heute Tourismus und prekäre Zuwanderung. Einige der Gassen sind belebt und voller Touristen, andere werden von Besuchern wie auch Einheimischen gemieden. Die befürchtete Gentrifizierung ist ausgeblieben, aber auch die von den Medien vielfach beschworene Migrationskrise. Der Arbeitsmarkt bietet kaum noch Möglichkeiten für die Zuwanderer. Die Wirtschaftskrise, in der die Stadt seit vielen Jahren steckt, bringt so auch eine Verlangsamung der Zuwanderung. Die bis 2017 links regierte Stadt begegnete den Zuwanderern unaufgeregt und oft sogar solidarisch. So unterstützte die katholische Kirche in ihren Gemeinden die Arbeit mit den MigrantInnen und einer der Pater, der sich für die Armen in der Altstadt einsetzte, Don Andrea Gallo, genoss hohes Ansehen in der Stadt und auch über die Stadt hinaus. Die Stadtverwaltung arbeitete eng mit den zivilgesellschaftlichen und Freiwilligenorganisationen zusammen. Bis auf vereinzelte Auseinandersetzungen in der Nähe von Unterkünften gab es keine besonderen Vorkommnisse durch den Zuzug von MigrantInnen (Giordanella, 2016 sowie Interviews). Im folgenden Abschnitt wird exemplarisch eine solche solidarische Initiative vorgestellt.

\subsection{Das Gegengift zur Krise: die Herstellung von Normalität}

Im Zuge der Flüchtlingskrise 2015 entstand auf Initiative der Vereinigung Migrantes in den Ruinen des leerstehenden ehe- 
maligen Krankenhauses „San Raffaele“ die Initiative „Campus La Coronata“. Ursprünglich 2013 von Pater Don Giacomo angedacht als ein Übergangsheim für ehemalige Gefängnisinsassen, führte die Flüchtlingskrise dazu, dass überraschend auch Menschen in Norditalien aufgenommen werden sollten. Im Jahr 2016 kamen die ersten 50 MigrantInnen in der Stadt an. Das ehemals heruntergekommene Areal um das Krankenhaus sollte nun als Ausgangspunkt für etwas, das mehr war als die Grundversorgung (Schlafen, Essen, Kleidung), dienen. Es war als gelebte Solidarität konzipiert, wie sich einer der Begründer ausdrückte. Die Kooperative liegt etwa $7 \mathrm{~km}$ vom Zentrum entfernt auf einer Anhöhe. Geleitet wird das Projekt vom Präsidenten der Initiative „Un'altra storia“, einem Ehrenamtlichen und einem Pater. Sie reagierten mit ihrem Projekt zunächst auf die humanitäre Krise. Sie begründen ihr Vorgehen vor allem auch mit der institutionellen Krise des italienischen Staates. Der hat weder die Finanzmittel, die Menschen zurück in die Heimatländer zu schicken, noch eine funktionierende Polizei zur Verfügung. Anders als in Deutschland stellt sich damit gar nicht die Frage, ob es zu einer Abschiebung der MigrantInnen kommen kann:

Die Migranten haben alles versucht, sie wurden abgelehnt, sie sind dennoch im Land. Auch das ist eine schreiende Lüge: alle Medien lügen hier. Wenn man sagt, geh in dein Land zurück und ich lege meine Hände in den Schoß, das ist eine Lüge. Man weiß, dass man ihnen das Geld geben muss, um nach Hause zu fahren. Wie sollen wir das denn bezahlen? Italien hat nicht mal genug Geld, um das Benzin für die Polizeiautos zu bezahlen. Da fährt mal ein Streifenwagen, der nächste schon nicht. Oder die Länder mit denen es keine Reziprozitätsabkommen gibt, da haben die Migranten dann plötzlich keine Dokumente mehr. Das erste, was die Migranten wegwerfen, ist der Pass, weil sie wissen, dass sie sonst zurückgeschickt würden. Wir haben nicht das Geld, die Leute zurückzuschicken, zwei Polizisten zur Begleitung anzustellen. Wie kommt man eigentlich überhaupt darauf, dass die Leute, die mühsam von einem Land weggegangen sind, zurückwollen?

Die meisten MigrantInnen wollten in der Tat weiter nach Nordwesteuropa mit den florierenden Arbeitsmärkten und sahen Italien als Transitland an (Borri, 2016). Dies erschwerte die Integration zusätzlich. Denn die wenigsten sahen ein, wozu sie in einem Land mit einem für sie kaum zugänglichen Arbeitsmarkt und in dem sie sich daher nur übergangsweise aufhalten wollen, Sprachkenntnisse erwerben sollten. Meist war das eigentliche Ziel ihres Migrationsprojektes, in Nordwesteuropa Geld zu verdienen und dieses nach Hause zu schicken. Der Aufenthalt in Italien war für sie nur Teil des Transits.

Der Ansatz des Projektes „Campus“ stellte sich als eine Mischung aus „Top-down“ - und „Bottom-up“ -Ansätzen

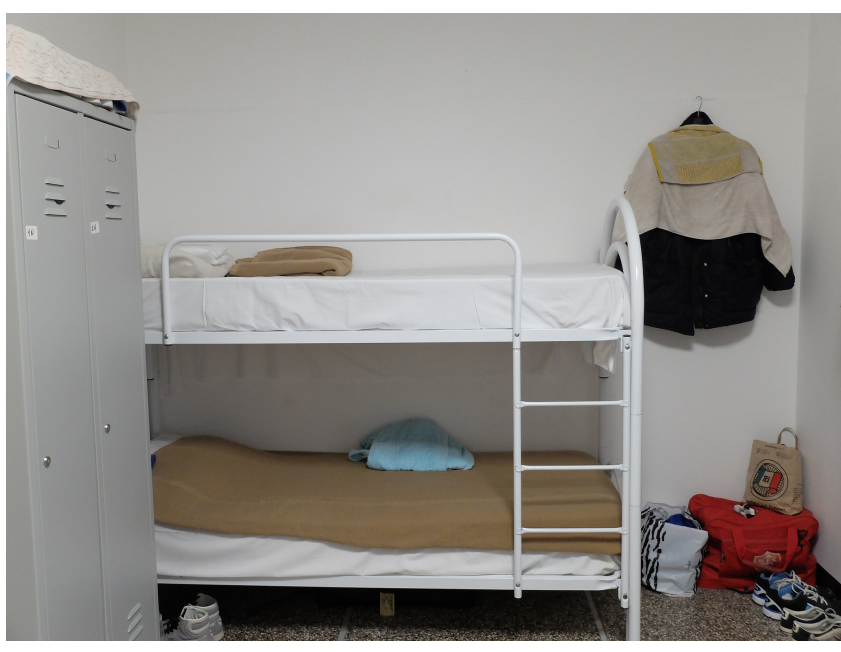

Abb. 1. Innenansicht eines Vierbettzimmers.

dar. Top-down, indem Gelder aus dem SPRAR-System bezogen wurden und dadurch dass die Kommune Genua das bis dahin brachliegende Gelände des ehemaligen Krankenhauses zur Verfügung stellte - während die Region Ligurien das Vorhaben blockierte. Kern des Projektes war die Renovierung und der Ausbau eines Campus in Eigenarbeit. Im Modus eines learning by doing entstanden in der Ruine neue Räume, das benötigte Baumaterial wurde von der Diözese und anderen Trägern gespendet. Die Initiatoren setzten auf eine Einbeziehung des lokalen Umfeldes - so wie dies auch im SPRAR-Programm vorgesehen war. Besonders die umliegenden Handwerksbetriebe wurden angesprochen und beteiligt, nach und nach sollten die verschiedenen zivilgesellschaftlichen Kräfte der Stadt Genua eingebunden werden. Das in „La Coronata“ erprobte Modell setzte auf ein umfassendes Programm nachhaltiger Integration. Tagsüber, von Montag bis Freitag von 09.00 bis $18.00 \mathrm{Uhr}$, lernten die Migranten auf dem Campus. Es gab obligatorische Fächer, sechs Stunden die Woche und außerdem Ausbildungseinheiten zum Schneider, Elektriker, Maurer, Installateur, im Bauwesen, in der Landwirtschaft, Fotografie und Musik. Entstanden war so ein Gelände, zu dem täglich 255 MigrantInnen zu Ausbildungszwecken aus der Stadt kamen. Es wurden Sprachkurse angeboten, die mit einem Zertifikat der Università per Stranieri (Perugia) abgeschlossen werden konnten. Es gab einen Bolzplatz, einen Bocciaplatz und eine Mensa, ein medizinisches Versorgungszentrum war in Planung. 80 Personen wohnten dort in 12-Bett-Zimmern. Nach und nach sollten sie zu 4-Bett-Zimmern umgebaut werden (Abb. 1).

Inzwischen ist es auch gelungen, auf kultureller Ebene etwas anzubieten. So fand ein Tag der offenen Tür statt, außerdem vernetzte sich das Projekt mit einem Poesiefestival (siehe Abb. 2). Das Projekt agierte weiterhin „bottom-up“, indem die genuesische Bevölkerung aktiv einbezogen wurde. Es war das genau „Ungeplante“, das zum eigentlichen Motor 
$24^{\circ}$ Festival Internazionale di Poesia di Genova - Parole Spalancate 2018

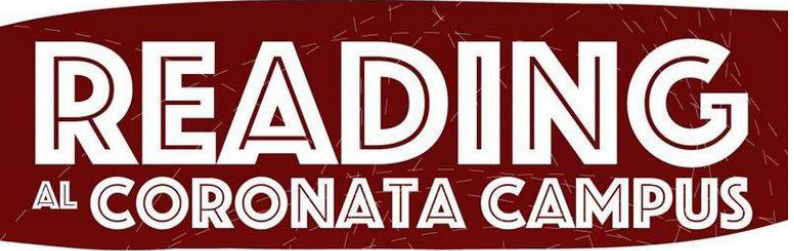

7 giugno 2018 - via Coronata, 100 - Genova Cornigliano - dalle 16.30 alle 18.00

Abb. 2. Teilnahme am Literaturfestival Parole Spalancate.

des Handelns wurde. Die Veranstalter erklärten das Prinzip, das quer zu den herkömmlichen Ansätzen der Planungspolitik verlief und dessenwichtigster Bestandteil kapillare Praxen der Vernetzung waren:

Es ist die Kunst sich zu arrangieren (ital: l'arte di arrangiarsi), also von unten was zu machen, und da muss ich sagen, dass wir gut sind - wir haben keine Exzellenz aufzubieten, aber das ist, glaube ich, schon etwas. In Italien haben wir viele Spezialisierungen, aber die Kunst, sich zu arrangieren, das ist etwas, was wir können.

Die jungen Männer hielten sich für 12, höchstens bis zu 18 Monate in dem Zentrum auf. Zum Zeitpunkt unserer Befragung stammten sie aus Mali, Nigeria, Senegal, Ghana, Gambia, Elfenbeinküste, aber auch Bangladesch, Pakistan und Afghanistan. Italien, speziell die Stadt Genua, war vielleicht nicht das von ihnen erträumte Ziel. Doch die Organisatoren des Campus akzeptierten, dass sie aus ihrem Heimatland wegwollten und ihren Träumen folgten. Sie nahmen in Kauf, dass die Jugendlichen Italien eventuell wieder verlassen mussten. Sie argumentierten, dass diese dann trotzdem etwas gelernt haben würden, das ihnen weiterhelfen könnte. Die Stärke des eigenen Ansatzes wurde indes nicht so sehr in der Ausbildung, sondern in der Integrationsfähigkeit durch Gastfreundlichkeit gesehen:

Und das ist das Geheimnis der Integration von unten, das muss nicht formal sein, nicht institutionell. Das ist vielleicht nicht ausreichend, aber es ist effizient. Am Ende ist das die wirkliche Integration. Die Leute merken, dass ihnen nicht die Tür vor der Nase zugemacht wird. Es sind bescheidene Ansätze, doch wir sind stolz darauf.

\section{Fazit und Ausblick}

Die europäische Migrationskrise ist Ergebnis des Zusammenwirkens mehrerer langjähriger Krisen auf ganz verschiedenen Handlungsebenen. Der hier gewählte analytische $\mathrm{Zu}-$ gang über eine sozialgeographische Betrachtung, die die verschiedenen Handlungsebenen getrennt analysiert, zeigt auf einer generellen Ebene, dass die „Migrationskrise“ Bestandteil und Ausdruck eines umfassenderen gesellschaftlichen Transformationsprozesses durch Migration und Mobilität ist. Es kommt zu einem Mehr an Wohlstandsmobilität (bei den EU-Bürgern) und zu Exklusion (bei den MigrantInnen). Die Zahlen deuten im Falle Italiens auf eine Normalisierung der Krise auf einem hohen Niveau hin: die Zahlen zu den Toten im Mittelmeer nehmen zwar ab, doch Migrationsindustrien sorgen für steten Nachschub an MigrantInnen nach Italien aus den vulnerablen Herkunftsländern. Dort wurde Migration für viele Menschen zu ihrer Coping-Strategie im Umgang mit der strukturellen Krise. Für die MigrantInnen selbst wurde „Europa“ das Ziel ihres Projektes, nicht das krisengeschüttelte Italien mit seinem widersprüchlichen und hochbürokratisierten Aufnahmesystem und einem schlecht zahlenden informellen Arbeitsmarkt. Das italienische System der Hilfsangebote und Aufnahmezentren (SPRAR) war durch die Auslagerung auf einzelne NGOs der staatlichen Kontrolle weitgehend entzogen - obwohl die entsprechenden Zentren durch den Staat finanziert wurden. Die Städte nahmen in der Bearbeitung der Migrationskrise durch die unzulänglichen nationalen Politiken eine besonders starke Position ein und agierten vielfach proaktiv durch die lokale Bewältigung der Migrationskrise. Das angeführte Beispiel der Stadt Genua reflektierte so auch die starke Rolle der Städte in dieser nationalstaatlichen Krise. Was zunächst als wenig formalisierte städtische Integrationspolitik erschien, wurde bei genauerer Betrachtung zum Gegengift gegen die Krisenrhetorik: Unaufgeregtheit und Normalisierung, pragmatische Versuche etwas für die Menschen in dieser schwierigen Situation zu tun - wie es sich in den Aktionen der Stadtverwaltung „unter dem Radar“ manifestierte. Die hier betrachtete SPRAR-Initiative „La Coronata“ zeigt, wie wichtig die jeweils bestehenden zivilgesellschaftlichen Praxen sind, um der Migrationskrise etwas entgegenzusetzten und wie sehr sich die Akteure darüber bewusst waren, dass Italien nicht die Mittel hätte, MigrantInnen zurückzuschicken oder abzuschieben, wie dies beispielsweise in Deutschland der Fall ist. Diese Erkenntnis lenkte die Handlungslogiken der Helfenden so auch in Richtung einer fragilen Integration. Die Organisatoren des hier betrachteten Fallbeispiels machten sich die erprobten kapillaren Praxen zunutze, um das Umfeld in die Integrationsbemühungen einzubinden. Es demonstrierte zugleich die Ohnmacht vieler südeuropäischer Städte, die immer dann aktiv werden müssen, wenn der informelle Arbeitsmarkt die vielen Arbeiter nicht mehr braucht und auf die Straße setzt. Die behauptete und die gelebte Migrationskrise, dies sollte hier herausgearbeitet werden, bestehen nebeneinander. Wer auf eindeutige, lineare und widerspruchsfreie Interpretationen der Migrationskrise im Falle Italiens gehofft hatte, muss leider enttäuscht werden: Was wir heute als europäische Migrationskrise in Italien erkennen, ist ein Konglomerat aus Vulnerabilitätskrisen in den Herkunftsländern der Migration, dem mobility paradox und der nationalstaatlichen Krise in Italien, die die Städte in Handlungsnot bringt und 
sich in eine Kontrollkrise übersetzt. Die in der Migrationskrise aufeinandertreffenden widersprüchlichen Handlungslogiken begründen sich auf den verschiedenen Reichweiten der räumlichen Definitionsmacht der Akteure. Während die MigrantInnen selbst angesichts nicht mehr vorhandener legaler Migrationswege nach Europa den Weg über Italien nach Nord- und Westeuropa als Lösung ihrer Existenzkrise in den Herkunftsländern ansahen und ihre räumliche Definitionsmacht so maximal ausnutzten, begab sich der italienische Staat in eine Dauerkrise, indem er Ausnahmelösungen (Unterbringung in Zentren unterschiedlichsten Typs) zu Dauerlösungen machte. Die Städte wiederum beteiligten sich an der Krisenproduktion dadurch, dass sie die Migration größtenteils als Notfall betrachteten und keine längerfristigen Integrationskonzepte entwarfen, obwohl sie wissen, dass die MigrantInnen vor Ort bleiben (müssen) und dass sie es mit einer humanitären Krise zu tun haben. Zum Teil wirkten dagegen die zivilgesellschaftlich organisierten Initiativen des SPRAR-Systems auf eine Eingliederung der MigrantInnen hin.

Datenverfügbarkeit. Die verwendeten Daten sind nicht allgemein zugänglich.

Interessenkonflikt. Die Autorin erklärt, dass kein Interessenkonflikt besteht.

Danksagung. Ich bedanke mich bei meinen geduldigen Gesprächspartnern für die vertrauensvollen Gespräche und bei Annalena Morra für ihre wunderbare Unterstützung während zweier Aufenthalte in Genua. Außerdem danke ich drei anonymen Gutachtern und dem Herausgeber für die hilfreichen Kommentierungen.

Begutachtung. This paper was edited by Benedikt Korf and reviewed by three anonymous referees.

\section{Literatur}

Adaaven, S. A.: Changing Reproductive Behavior and Migration in Response to Environmental Change: Evidence from Rural Northern Ghana, in: Environmental change, adaptation and migration: Bringing in the region, Herausgeber: Hillmann, F., Pahl, M., Rafflenbeul, B., and Sterly, H., Palgrave Macmillan, Basingstoke, UK, 203-222, 2015.

Agamben, G.: Homo sacer: Die souveräne Macht und das nackte Leben, Suhrkamp, Frankfurt am Main, Deutschland, 211 Seiten, 2002.

Alpes, M. J.: Unravelling the legal consciousness of deportation policies through women's bushfalling narratives in Anglophone Cameroon, in: Trajectories and Imaginaries in Migration: The Migrant Actor in Transnational Space, Herausgeber: Hillmann, F., van Naerssen, T., and Spaan, E., Studies in migration and diaspora, Routledge, Abingdon, Oxon, New York, USA, 77-91, 2019.

Ambrosini, M.: Irregular immigration in Southern Europe: Actors, dynamics and governance, Migration, diasporas and citizenship, Palgrave Macmillan, Cham, Schweiz, 2018.

Asselin, O., Dureau, F., Fonseca, L., Giroud, M., Hamadi, A., Kohlbacher, J., Lindo, F., Malheiros, J., Marcadet, Y., und Reeger, U.: Social Integration of Immigrants with Special Reference to the Local and Spatial Dimension, in: The dynamics of international migration and settlement in Europe. A State of the Art, Herausgeber: Penninx, R., Berger, M., Kraal, K., Amsterdam, Niederlande, 133-170, 2006.

Awumbila, M., Deshingkar, P., Kandilige, L., Teye, J. K., and Setrana, M.: Brokerage in migrant domestic work in Ghana: complex social relations and mixed outcomes, Brighton, UK, Migrating out of Poverty RPC Working Paper, 47, 2016.

Benigni, F. and Pierdicca, M.: Keep Moving! Strategien der Wegmobilisierung als Teil des italienischen Migrationsmanagements, TRANSIT, 10, 1-13, 2016.

Borri, G.: "Mobilità" intra-europea: il caso dei movimenti di ritorno a Torino di migranti titolari di protezione umanitaria, Mondi Migranti, 61-82, https://doi.org/10.3280/MM2016-001004, 2016.

Campesi, G.: Between containment, confinement and dispersal: The evolution of the Italian reception system before and after the "refugee crisis", Journal of Modern Italian Studies, 23, 490-506, https://doi.org/10.1080/1354571X.2018.1501995, 2018.

Campomori, F. and Caponio, T.: Immigrant integration policymaking in Italy: regional policies in a multi-level governance perspective, Int. Rev. Adm. Sci., 83.2., 303-321, 2017.

Caneva, E.: The integration of migrants in Italy: an overview of policy instruments and actors, INTERACT RR 2014/05, Robert Schuman Centre for Advanced Studies, San Domenico di Fiesole(FI): European University Institute, Florenz, Italien, 2014.

Caponio, T. and Cappiali, T. M.: Italian Migration Policies in Times of Crisis: The Policy Gap Reconsidered, S. Eur. Soc. Polit., 23, 115-132, https://doi.org/10.1080/13608746.2018.1435351, 2018.

Carmagnani, M. and Pastore, F.: Capire le migrazioni nell'epoca dell'incertezza., in: Migrazioni e integrazione in Italia tra continuità e cambiamento: Atti del Convegno tenuto presso la Fondazione Luigi Einaudi (Torino 6-7 ottobre 2016), Herausgeber: Carmagnani, M., and Pastore, F., Studi, 56, Olschki, Firenze, Italien, 2018

Castelli Gattinara, P.: The "refugee crisis" in Italy as a crisis of legitimacy, Contemporary Italian Politics, 9, 318-331, https://doi.org/10.1080/23248823.2017.1388639, 2017.

Chambers, R.: Editorial Introduction: Vulnerability, Coping and Policy, IDS Bull., 20, 1-7, https://doi.org/10.1111/j.17595436.1989.mp20002001.x, 1989.

Cuttitta, P.: Delocalization, Humanitarianism and Human Rights. The Mediterranean Border between Exclusion and Inclusion, Antipode, 50, 783-803, 2018.

Finotelli, C. and Echeverría, G.: So close but yet so far?: Labour Migration Governance in Italy and Spain, Int. Migr., 55, 39-51, https://doi.org/10.1111/imig.12362, 2017.

Fontanari, E.: Soggettività en transit. (Im)mobilità dei rifugiati in Europa tra sistemi di controllo e pratiche quotidiane di attraversamento dei confini, in: Incursioni. Rifugiati in transito attraver- 
so l'Europa, Hg. von Chiara Marchetti e Michele Manocchi, in: Rivista Mondo migranti, Genova, Italien, 39-60, 2016.

Fratesi, U. and Percoco, M.: Selective Migration, Regional Growth and Convergence: Evidence from Italy, Reg. Stud., 48, 16501668, https://doi.org/10.1080/00343404.2013.843162, 2014.

Gammeltoft-Hansen, T. and Nyberg Sørensen, N. (Hrsg.): The migration industry and the commercialization of international migration, Routledge global institutions series, 0069, Routledge, London, UK and New York, USA, xviii, 278, 2013.

Giordanella, N.: Profughi, il sistema dell'accoglienza a Genova. Numeri, criticità e prospettive, online aufrufbar: http://genova.erasuperba.it/notizie-genova/ profughi-accoglienza-genova-2016 (letzter Zugriff: 18 July 2019), 2016.

Glick Schiller, N. and Çağlar, A. (Hrsg.): Locating Migration. Rescaling Cities and Migrants, Ithaka, Cornell University press, Ithaca, USA, 2011.

Greck, R.: Schaffen wir das?: Frames im medialen Diskurs zur Flüchtlingskrise in der deutschen Regionalpresse des Jahres 2015 und ihre Lösungsorientierung, Publizistik, 63, 359-382, https://doi.org/10.1007/s11616-018-0437-x, 2018.

Greenhill, K. M.: Open Arms Behind Barred Doors: Fear, Hypocrisy and Policy Schizophrenia in the European Migration Crisis, Eur. Law J., 22, 317-332, https://doi.org/10.1111/eulj.12179, 2016.

Greussing, E. and Boomgaarden, H. G.: Shifting the refugee narrative?: An automated frame analysis of Europe's 2015 refugee crisis, J. Ethn. Migr. Stud., 43, 1749-1774, https://doi.org/10.1080/1369183X.2017.1282813, 2017.

Hampshire, J.: Europe's Migration Crisis, Political Insight, 6, 8-11, https://doi.org/10.1111/2041-9066.12106, 2015.

Hillmann, F.: Migration - eine Einführung aus sozialgeographischer Perspektive. Reihe Sozialgeographie kompakt, Steiner Verlag, Stuttgart, Deutschland, 2016.

Hillmann, F. and Calbet i Elias, L.: Zwischen Realitätsverweigerung und Pragmatismus: Migration-led regeneration in Genua und Manchester, Raumforschung und Raumordnung, 77, 1-17, https://doi.org/10.2478/rara-2019-0038, 2019.

Hillmann, F. and Ziegelmayer, U.: Environmental Change and Migration in Coastal Regions: Examples from Ghana and Indonesia, Erde, 147, 119-138, https://doi.org/10.12854/erde-147-9, 2016.

Hillmann, F., van Naerssen, T., and Spaan, E. (Hrsg.): Trajectories and Imaginaries in Migration: The Migrant Actor in Transnational Space, Studies in migration and diaspora, Routledge, Abingdon, Oxon, New York, USA, 2019.

Jarawura, F. X. and Smith, L.: Finding the Right Path: Climate Change and Migration in Northern Ghana, in: Environmental change, adaptation and migration: Bringing in the region, Herausgeber: Hillmann, F., Pahl, M., Rafflenbeul, B., and Sterly, H., Palgrave Macmillan, Basingstoke, UK, 245-266, 2015.

Kleist, N. and Thorsen, D. (Hrsg.): Hope and uncertainty in contemporary African migration, Routledge studies in anthropology, 30, Routledge, New York, USA, London, UK, xiii, 199, 2017.

Klepp, S.: Europa zwischen Grenzkontrolle und Flüchlingsschutz: Eine Ethnographie der Seegrenze auf dem Mittelmeer, transcript, Bielefeld, Deutschland, 424 Seiten, 2011.
Klepp, S.: Haftzentren für Migrant_innen in Italien - der andauernde Ausnahmezustand, SozProb, 26, 171-188, https://doi.org/10.1007/s41059-015-0007-7, 2015.

Lucht, H.: Death of a Gin Salesman: Hope and Despair among Ghanaian Migrants and Deportees Stranded in Niger, in: Hope and uncertainty in contemporary African migration, Herausgeber: Kleist, N. and Thorsen, D., Routledge studies in anthropology, 30, Routledge, New York, USA, London, UK, 154-172, 2017.

Manuh, T.: An 11th Region of Ghana?: Ghanaians Abroad, Inaugural Lecture, Accra, Ghana, 2006.

Ministero Dell'Interno (Hrsg.): Piano nazionale d'integrazione dei titolari di protezione internazionale, Dipartimento per le Libertà Civili e l'Immigrazione, Rom, Italien, 2017.

Panizzon, M. and van Riemsdijk, M.: Introduction to Special issue: "migration governance in an era of large movements: a multi-level approach", J. Ethn. Migr. Stud., 45, 1225-1241, https://doi.org/10.1080/1369183X.2018.1441600, 2018.

Plambech, S.: Becky is dead, in: OpenDemocracy, online aufrufbar: https://www.opendemocracy.net/beyondslavery/sine-plambech/ becky-is-dead, letzter Zugriff: 31 October 2017.

Pries, L.: Die Transnationalisierung der sozialen Welt: Sozialräume jenseits von Nationalgesellschaften, Edition Suhrkamp, Frankfurt am Main, Deutschland, 2007.

Rigo, E.: Migration, knowledge production and the humanitarian agenda in times of crisis, J. Mod. Ital. Stud., 23, 507-521, https://doi.org/10.1080/1354571X.2018.1501996, 2018.

Schapendonk, J.: Stuck between the desert and the Sea: The immobility of Sub-Saharan African "Transit Migrants" in Morocco, in: Rethinking global migration: Practices, policies and discourses in the European neighbourhood, Herausgeber: RittersbergerTiliç, H., METU, KORA, Ankara, Türkei, 129-141, 2008.

Sen, A.: Poverty and famines: An essay on entitlement and deprivation, Oxford Univ. Press, Oxford, UK, ix, 257, 1981.

Sheller, M.: Mobility Justice. The Politics of Movements in an Age of Extremes, Brooklyn and London, UK, 2018.

van der Geest, K.: North-South Migration in Ghana: What Role for the Environment?, Int. Migr., 49, e69-e94, https://doi.org/10.1111/j.1468-2435.2010.00645.x, 2011.

Vowe, G.: Politische Kommunikation in der Migrationskrise: Der strukturelle Wandel der Kommunikation als Herausforderung für Politik und Wissenschaft, Publizistik, 4, 431-440, 2016.

Watts, M. J. and Bohle, H. G.: The space of vulnerability: The causal structure of hunger and famine, Prog. Hum. Geog., 17, 43-67, https://doi.org/10.1177/030913259301700103, 1993.

Zeit online: Mehr als 1.400 Tote im Mittelmeer, online aufrufbar: https://www.zeit.de/gesellschaft/zeitgeschehen/2018-07/ fluechtlinge-mittelmeer-flucht-tote-2018-organisation-fuer-migration, letzter Zugriff: 18 July 2018.

Zincone, G.: The case of Italy, in: Migration policymaking in Europe: The dynamics of actors and contexts in past and present, Herausgeber: Zincone, G., Penninx, R., and Borkert, M., IMISCOE research, Amsterdam University Press, Amsterdam, Niederlande, 247-290, 2011. 